\title{
RISCOS OCUPACIONAIS E ALTERAÇÕES DE SAÚDE ENTRE TRABALHADORES DE ENFERMAGEM BRASILEIROS DE UNIDADES DE URGÊNCIA E EMERGÊNCIA ${ }^{1}$
}

\author{
OCCUPATIONAL HAZARDS AND CHANGES OF HEALTH AMONG \\ BRAZILIAN PROFESSIONALS NURSING FROM URGENCY AND \\ EMERGENCY UNITS
}

\section{RIESGOS OCUPACIONALES Y ALTERACIONES DE SALUD ENTRE TRABAJADORES BRASILENÕS DE ENFERMERÍA EN UNIDADES DE URGENCIA Y EMERGENCIA}

\author{
Rita de Cássia de Marchi Barcellos Dalri* \\ Maria LÚcia do Carmo Cruz Robazzi ${ }^{* *}$ \\ Luiz Almeida DA SiLva ${ }^{* * *}$
}

\begin{abstract}
RESUMO
Objetivo: identificar os riscos ocupacionais existentes no ambiente de trabalho em Unidades Públicas de Urgência e Emergência e as alterações de saúde apresentadas pelos trabalhadores de enfermagem que ali atuam. Método: estudo não experimental, com análise quantitativa dos dados, delineamento transversal, realizado em duas Unidades de uma cidade do Estado de Minas Gerais - Brasil, em 2006. Tornaram-se sujeitos 64 trabalhadores entre Enfermeiros, Técnicos e Auxiliares de Enfermagem, correspondendo a 55,17\% do total; para a coleta de dados utilizou-se um questionário semi-estruturado. Resultados: os sujeitos, em sua maioria são mulheres, casadas, com idade entre 20 e 59 anos, auxiliares de enfermagem, concursados, com carga horária de trabalho semanal entre 44 e $136 \mathrm{~h}$. Dentre os Riscos Ocupacionais identificados, os psicossociais foram os mais citados e as alterações de saúde mais encontradas foram, algias, cansaço mental e estresse, distúrbios do sono, alterações cardiovasculares e processos infecciosos que em sua maioria podem ter sido provocados pelos riscos identificados. Conclusão: o ambiente de trabalho nas Unidades de Urgência e Emergência é evidentemente estressante, o que justifica a identificação dos Riscos Ocupacionais e a maioria das alterações de saúde encontradas.
\end{abstract}

Palavras chave: Equipe de enfermagem, enfermagem em emergência, riscos ocupacionais, saúde do trabalhador, trabalho.

\footnotetext{
ABSTRACT

Objective: To identify the occupational hazards existing in Urgency and Emergency Public Units environment, and the related health problems shown by those professionals working there. Method: Non-experimental study with data quantitative analysis, transverse outlining, carried out at two units in a city in Minas Gerais state Brazil in 2006. Sixty four professionals were used as subjects for the analysis, including nurses, nursing technicians and assistants, which correspond to $55.17 \%$ of the nursing professionals as a whole; for the collected data a semi-structured questionnaire was used. Results: The majority of subjects are women, aged between 20 and

${ }^{1}$ Dissertação de mestrado.

* Enfermeira. Mestrando pela Escola de Enfermagem de Ribeirão Preto da Universidade de São Paulo. Brasil. E-mail: ritacmbdalri@bol.com.br

${ }^{* *}$ Enfermeira. Professora Titular da Escola de Enfermagem de Ribeirão Preto da Universidade de São Paulo. Brasil.

E-mail: avrmlccr@eerp.usp.br

${ }^{* * *}$ Enfermeiro. Mestrando pela Escola de Enfermagem de Ribeirão Preto da Universidade de São Paulo. Brasil.

E-mail: enferluiz@yahoo.com.br
} 
59 years, nursing assistants holding permanent job positions with weekly workload of 44 and $136 \mathrm{~h}$. Among the identified occupational hazards the psychosocial were the most quoted ones and the most common health alterations were pains, mental disorder and stress, sleep disorder, cardiovascular alterations and infectious processes, which mostly, coincide with the identified risks. Conclusion: The work environment in Urgency and Emergency Units is clearly stressing, which justifies the identification of occupational hazards in most of health problems that have been found.

Key words: Nursing team, emergency nursing, occupational hazard, occupational health, work.

\section{RESUMEN}

Objetivo: Identificar los riesgos ocupacionales existentes en el ambiente de trabajo de las Unidades Públicas de Urgencia y Emergencia y las alteraciones de salud presentadas por los trabajadores que allí actúan. Método: Estudio no experimental, con análisis cuantitativa de los datos, delineamiento transversal, realizado en dos unidades de una ciudad del Estado de Minas Gerais, Brasil, en 2006. Participaron en el estudio 64 trabajadores entre enfermeros, técnicos y auxiliares de enfermería, correspondiendo a 55,17\% del total; para la recopilación de los datos se utilizó un cuestionario semiestructurado. Resultados: La mayoría de los sujetos son mujeres casadas con edad entre 20 y 59 años, auxiliares de enfermería, concursados, con carga horaria de trabajo semanal entre 44 y $136 \mathrm{~h}$. Entre los riesgos ocupacionales identificados, los psicosociales fueron los más citados y las alteraciones de salud más encontradas fueron los dolores, cansancio mental y estrés, disturbios del sueño, alteraciones cardiovasculares y procesos infecciosos que en su mayoría coinciden con los riesgos identificados. Conclusión: El ambiente de trabajo en las Unidades de Urgencia y Emergencia es evidentemente estresante, lo que justifica la identificación de los riesgos ocupacionales y la mayoría de las alteraciones de salud encontradas.

Palabras clave: Equipo de enfermería, enfermería de emergencia, riesgos ocupacionales, salud del trabajador, trabajo.

Fecha recepción: 06/01/09 Fecha aceptación: 07/05/10

\section{INTRODUÇÃO}

Os processos laborativo e o de produção, estabelecidos no contexto do trabalho e nos quais o trabalhador participa como agente podem-se compor em fatores determinantes para o desgaste de sua saúde (1). A constante mudança no mundo do trabalho que vem sendo imposta aos trabalhadores e a capacidade dos mesmos em ajustar-se a ela, podem lhes proporcionar crescentes incertezas, insatisfação generalizada com o modo de vida, aflorando sentimentos de tédio, angústia, sofrimento, mas também vivências de prazer (2).

Entre as várias categorias de trabalhadores, existem os de enfermagem, que no Brasil encontram-se segmentados em três grupos: auxiliares de enfermagem, técnicos de enfermagem e enfermeiros, respectivamente com formação de nível fundamental, médio e superior (3). Esses trabalhadores possuem como função peculiar prestar assistência ao indivíduo sadio ou doente, família ou comunidade, no desempenho de atividades para promover, manter ou recuperar a saúde (4). $\mathrm{O}$ enfermeiro atua interagindo com os demais trabalhadores inseridos no sistema de cuidados em saúde, nas suas relações/interações/associações para o processo de cuidar da vida e da morte. O trabalho da equipe de enfermagem é heterogêneo e hierarquizado; enquanto no processo de cuidar, os agentes preponderantes são os auxiliares e técnicos de enfermagem, no processo de administrar, são os enfermeiros (5). O campo de atuação profissional é variado incluindo hospitais, ambulatórios, escolas, empresas, forças armadas, entre outros, considerando-se também o trabalho em unidades de urgência e 
emergência (UUE). Tais unidades realizam o primeiro atendimento à maioria das ocorrências médicas, devendo apresentar caráter resolutivo para os casos de menor gravidade que, na prática, costumam representar um percentual significativo dos pacientes atendidos, encaminhamento dos casos mais graves para internação hospitalar, para cirurgia eletiva ou para o atendimento médico especializado indicado para o cliente (6). A progressiva desestruturação da assistência à Saúde Pública no Brasil ocorrida através dos anos, devido à falta de planejamento, dos desvios de verbas e outras causas, trouxe a deterioração das instituições de saúde e o desgaste de seus recursos humanos. Uma crescente demanda e precárias condições de atendimento resultaram em diminuição no padrão assistencial por parte dos prontos socorros e das demais UUE (7).

A inadequação das condições de trabalho nestas unidades, quando estas funcionam de forma improvisada ou indesejável é caracterizada pela maior exposição dos trabalhadores aos agentes de riscos ocupacionais ( $\mathrm{RO})$, os quais podem ser de origem biológica, química, física, psicossocial e situações anti-ergonômicas, incluindo os fatores de violência aos quais eles ficam expostos durante sua atividade laboral (8). Trabalhadores de enfermagem normalmente sujeitam-se aos vários agentes de RO, adoecem, acidentam-se e na maioria das vezes, não relacionam esses problemas à sua atividade laboral.

A violência ocupacional que pode ocorrer em seus ambientes de trabalho relaciona-se principalmente, aos indivíduos em situações críticas (toxicômanos, alcoólatras, vítimas de acidentes de trânsito, vítimas de acidentes por arma branca ou arma de fogo, entre outros) e esses ambientes de trabalho não estão equipados de forma a oferecer segurança aos seus trabalhadores (8). Além dessa condição perpetrada pela situação material e ambiental acrescida da realização de um trabalho insalubre; outra forma de violência do trabalho que vem se intensificando, especialmente com as recentes mudanças na organização laboral, é aquela ligada à carga e ritmo de execução das tarefas. O resultado disto é, como se poderia esperar, sofrimento psíquico e aumento de adoecimentos (9). O objetivo do presente estudo foi, então, identificar os RO existentes no ambiente de trabalho em UUE e as alterações de saúde apresentadas pelos trabalhadores de enfermagem que ali atuam.

\section{MATERIAL E MÉTODO}

Estudo não experimental, com análise quantitativa dos dados e delineamento transversal, obtido através de métodos de avaliação descritivos, utilizando o questionário como instrumento de coleta de dados. Este instrumento foi dividido em três partes, a saber, a primeira com questões direcionadas às variáveis sócio-demográficas e profissionais; a segunda com questões para a obtenção de dados sobre problemas de saúde e a terceira e última seção, com questões sobre os RO existentes no ambiente de trabalho. Esta coleta de dados foi realizada em duas UUE (UUE1 e UUE2) da Secretaria Municipal de Saúde (SMS) de uma cidade do interior de Minas Gerais, Brasil; em janeiro de 2006. O ingresso ao emprego na SMS acontece em sua maioria por meio de concurso público. Em ambas as unidades, foram identificados 116 trabalhadores, entre enfermeiros, auxiliares e técnicos de enfermagem.

Os critérios de inclusão adotados para a constituição do número de sujeitos do estudo foram: ser trabalhador de enfermagem atuante em UUE ou em outras unidades da SMS, mas que em dias de feriados, finais de semana ou turnos noturnos trabalha em tal unidade; aceitar participar da pesquisa; estar presente nas datas estipuladas para a coleta de dados e estar ausente por motivo de adoecimento relacionado ao trabalho. Foram excluídos os que estavam usufruindo férias 
e licenças (exceto licenças por problemas de saúde relacionados ao trabalho) e os ausentes na data estipulada para coleta de dados. Obedecidos tais critérios, constituiram-se sujeitos do estudo 64 trabalhadores, representando $55,17 \%$ em relação ao total, selecionados segundo amostragem de conveniência. Sabese que este tipo de amostragem é empregado quando se deseja obter informações de maneira mais rápida; tal procedimento ocorre quando a participação é voluntária ou os elementos da amostra são escolhidos por uma questão de conveniência (10).

Em relação aos procedimentos éticos, obedeceu-se ao preconizado na Resolução 196/96 do Ministério da Saúde do Brasil (11). A autorização para realização do estudo foi concedida pela SMS da cidade brasileira e a aprovação da pesquisa ocorreu junto ao Comitê de Ética em Pesquisa da Escola de Enfermagem de Ribeirão Preto da Universidade de São Paulo (Protocolo No 0618/2005).

As questões foram respondidas pelos sujeitos, em seu horário e postos de trabalho, mediante liberação da chefia imediata. Apenas uma trabalhadora que havia se submetido a uma cirurgia nos ombros foi entrevistada em seu domicílio. Antes de ser aplicado, o instrumento de coleta de dados foi validado por quatro especialistas na temática de Saúde do Trabalhador, para verificação de sua clareza, semântica, objetividade e conteú- do. Ocorreram algumas sugestões e após os ajustes necessários, foi efetuado um estudo piloto entre oito trabalhadores de enfermagem atuantes em outras UUE, permitindo aprimorá-lo.

As queixas à saúde citadas pelos sujeitos foram agrupadas conforme as classificações de diagnósticos médicos; assim todos os problemas de pele foram agrupados em alterações dermatológicas, os de cansaço, irritabilidade, esgotamento, em cansaço mental e estresse e assim por diante. Após a codificação apropriada de cada uma das variáveis, elaborou-se um questionário (codebook), bem como um banco de dados, empregando-se um aplicativo MS Excel XP.

O processo de validação por dupla alimentação foi realizado mediante duas digitações independentes utilizando-se duas planilhas. A análise estatística; uni e bi variada foi executada utilizando-se o Programa SPSS (Statistical Package for the Social Sciences), versão 15.0. Os dados obtidos foram comparados e discutidos com a literatura existente.

\section{RESULTADOS E DISCUSSÃO}

Algumas das características pessoais e profissionais dos trabalhadores de enfermagem, sujeitos do estudo, encontram-se a seguir.

Tabela 1. Distribuição de trabalhadores de enfermagem que atuam em duas UUE*, segundo suas características pessoais e profissionais, $2006(\mathrm{n}=64)$.

\begin{tabular}{lrrrrrr}
\hline Variáveis & \multicolumn{2}{c}{ UUE1 } & \multicolumn{2}{c}{ UUE2 } & \multicolumn{2}{c}{ TOTAL } \\
& f & $\%$ & \multicolumn{1}{c}{ f } & \multicolumn{1}{c}{$\%$} & \multicolumn{1}{c}{ f } & \multicolumn{1}{c}{$\%$} \\
\hline Sexo & 30 & 96.77 & 24 & 72.73 & 54 & 84.38 \\
Feminino & 1 & 3.23 & 9 & 27.27 & 10 & 15.62 \\
Masculino & 31 & 100.00 & 33 & 100.00 & 64 & 100.00 \\
Total & & & & & & \\
Estado civil & 9 & 29.03 & 13 & 39.39 & 22 & 34.38 \\
Solteiro & 16 & 51.61 & 14 & 42.42 & 30 & 46.87 \\
Casado & 4 & 12.90 & 5 & 15.15 & 9 & 14.06 \\
Divorciado & & & & & & \\
\hline
\end{tabular}


Riscos ocupacionais e alteraçốes de saúde entre trabalhadores de enfermagem brasileiros... / R. Barcellos D., M. Cruz R., L. Almeida da S.

Continuaçao Tabela 1.

\begin{tabular}{lrrrrrr}
\hline Viúvo & 2 & 6.45 & 1 & 3.03 & 3 & 4.68 \\
Total & 31 & 100.00 & 33 & 100.00 & 64 & 100.00 \\
Faixa etária & & & & & & \\
Média & 43 & - & 41 & - & 42 & - \\
Mínima & 21 & - & 20 & - & 20 & - \\
Máxima & 59 & - & 59 & - & 59 & - \\
Categoria profissional & & & & & & \\
Enfermeiro & 5 & 16.13 & 7 & 21.21 & 12 & 18.75 \\
Técnico de Enfermagem & 3 & & 4 & 12.12 & 7 & 10.94 \\
Auxiliar de Enfermagem & 23 & 74.19 & 22 & 66.67 & 45 & 70.31 \\
Total & 31 & 100.00 & 33 & 100.00 & 64 & 100.00 \\
Vínculo empregatício & & & & & & \\
Concursado & 26 & 83.87 & 24 & 72.73 & 50 & 78.13 \\
Contratado & 5 & 16.13 & 9 & 27.27 & 14 & 21.87 \\
Total & 31 & 100.00 & 33 & 100.00 & 64 & 100.00 \\
Carga horária semanal & & & & & & \\
Média & 58 & - & 62 & - & 64 & - \\
Mínima & 44 & - & 44 & - & 44 & - \\
Máxima & 136 & - & 114 & - & 136 & - \\
\hline
\end{tabular}

*UUE: Unidade de Urgência e Emergência

No que refere ao sexo, ambas as unidades apresentam a predominância do feminino $(84,4 \%)$, demonstrando que a enfermagem brasileira ainda continua sendo uma profissão essencialmente desenvolvida por mulheres, como vem ocorrendo ao longo da sua história. Esta realidade deve-se também aos baixos salários oferecidos para tais trabalhadores, dificultando para os homens, chefes de família optar por tal profissão (12). Nestas unidades prevalecem os sujeitos casados (46,9\%) e com média de faixa etária de 43 anos na UUE1 (as idades variaram de 21 a 59 anos) e de 41 anos na UUE2 (as idades variaram de 20 a 59 anos), evidenciando uma realidade muito próxima, em ambas. A maioria (70,3\%) é auxiliar de enfermagem e concursada $(78,1 \%)$; os demais trabalhadores $(21,9 \%)$ são contratados pelo regime brasileiro da Consolidação das Leis do Trabalho (CLT), tendo sido admitidos para o serviço sem aprovação em concurso público municipal.

Esse fato indica que a atuação dos trabalhadores ocorre em uma situação de estabi- lidade empregatícia e salários garantidos, o que sempre representou o emprego no Serviço Público no Brasil. Atualmente, os servidores públicos detêm o maior número dos trabalhadores da saúde no Brasil.

As horas semanais de trabalho variaram de 44 a 136; identificando-se uma média de $58 \mathrm{~h}$ semanais na UUE1 e de $62 \mathrm{~h}$ semanais na UUE2, o que representa horas de atividades extras. A média geral apresentada foi de $64 \mathrm{~h}$ de trabalho por semana, evidenciando a superioridade em relação às $36 \mathrm{~h}$ semanais permitidas atualmente pelo Conselho Federal de Enfermagem (COFEN) e excedendo em demasia, às $30 \mathrm{~h}$ semanais reivindicadas por este Conselho nos órgãos do Governo Federal. Para efeito do cálculo de horas para elaboração da escala mensal de serviço de enfermagem, atualmente a carga horária deverá ser de $36 \mathrm{~h}$ semanais para atividade assistencial; $40 \mathrm{~h}$ semanais para atividades administrativas e deve ser adaptado à carga horária estabelecida nos respectivos contratos de trabalho dos profissionais de enferma- 
gem (3). Ainda devido à baixa remuneração da categoria profissional, os trabalhadores de enfermagem buscam outros empregos para complementar sua renda familiar.
As alterações de saúde identificadas entre os trabalhadores de enfermagem encontramse apresentadas a seguir.

Tabela 2. Distribuição das alterações de saúde informadas por 64 trabalhadores de enfermagem que atuam em UUE*, $2006(n=169)$.

\begin{tabular}{lrrrrrr}
\hline \multirow{2}{*}{ Alterações de saúde } & \multicolumn{2}{c}{ UUE1 } & \multicolumn{2}{c}{ UUE2 } & \multicolumn{2}{c}{ TOTAL } \\
& f & \multicolumn{1}{c}{$\%$} & f & \multicolumn{1}{c}{$\%$} & \multicolumn{1}{c}{ f } & \multicolumn{1}{c}{$\%$} \\
\hline Algias & 37 & 44.05 & 30 & 35.29 & 67 & 39.64 \\
Cansaço mental/estresse & 10 & 11.90 & 15 & 17.65 & 25 & 14.79 \\
Cardiovasculares & 10 & 11.90 & 9 & 10.59 & 19 & 11.24 \\
Processos infecciosos & 5 & 5.95 & 8 & 9.41 & 13 & 7.69 \\
Distúrbios do sono & 5 & 5.95 & 5 & 5.88 & 10 & 5.92 \\
Intestinais & 3 & 3.57 & 2 & 2.35 & 5 & 2.96 \\
Respiratórias & - & - & 5 & 5.88 & 5 & 2.96 \\
De acuidade visual & 2 & 2.38 & 2 & 2.35 & 4 & 2.37 \\
Dermatológicas & 1 & 1.19 & 2 & 2.35 & 3 & 1.78 \\
Genito- urinárias & 1 & 1.19 & 1 & 1.18 & 2 & 1.18 \\
Ortopédicas & 1 & 1.19 & 1 & 1.18 & 2 & 1.18 \\
Hematológicas & - & - & 1 & 1.18 & 1 & 0.59 \\
Metabólicas & 1 & 1.19 & - & - & 1 & 0.59 \\
Neurológicas & 1 & 1.19 & - & - & 1 & 0.59 \\
Vasculares & 1 & 1.19 & - & - & 1 & 0.59 \\
Neoplasia & - & - & 1 & 1.18 & 1 & 0.59 \\
Outras & 6 & 7.14 & 3 & 3.53 & 9 & 5.33 \\
Total & 84 & 100.0 & 85 & 100.0 & 169 & 100.0 \\
\hline & & & & & &
\end{tabular}

*UUE: Unidade de Urgência e Emergência

${ }^{* *}$ Os trabalhadores informaram números variados de alterações de saúde

Entre as alterações de saúde, as algias foram as mais mencionadas, ou seja, 67 citações $(39,64 \%)$, seguidas de cansaço mental e estresse, com 25 citações (14,79\%) e, em menor percentual, por outras alterações, tais como as cardiovasculares, 19 citações (11,24\%), infecciosas, 13 (7,69\%), distúrbios do sono, $10(5,92 \%)$ e outros tipos de queixas, nove $(5,33 \%)$.

Dentre as queixas relacionadas às algias, as dores em membros inferiores (MMII) são as mais frequentes com 21 citações, provavelmente relacionadas às situações anti-ergonômicas (longos períodos de trabalho em pé) e elevada carga laboral diária. Enfatizase que vários sujeitos possuem atividades de trabalho em turnos diferentes e consequentemente, possuem período de sono e repouso deficientes. Além dessas dores, foram citadas as lombalgias (18 citações), as quais estão 
entre as queixas mais frequentes da população em geral. Representam grande parte das causas de concessão de auxílio doença previdenciário e de aposentadoria por invalidez (9). As cefaléias surgiram com 11 citações e a epigastralgia foi mencionada seis vezes; os sujeitos relacionam o surgimento dessas alterações com episódios de estresse eminente e cansaço físico extremo.

Cansaço mental e estresse surgem na sequência em segundo lugar. O estresse refere-se ao esgotamento que interfere na vida do indivíduo, mas não necessariamente na relação laboral. O trabalho da enfermagem propicia situações de estresse e causa sofrimento e adoecimento. Ainda que o trabalho possa ser uma fonte de prazer, de bem estar e de saúde quando existem condições favoráveis à livre utilização das habilidades e o seu controle pelos trabalhadores, por outro lado, pode ser origem de sofrimento psíquico quando desprovido de sentido, sem suporte social, não reconhecido ou em situações que se constitui em fonte de ameaça à integridade física e/ou psíquica (13).

Alterações cardiovasculares surgiram em terceiro lugar em frequência e dividiram-se em queixas de hipertensão arterial (HA), angina pectoris e taquicardia. A HA representa grave problema de saúde no país, não só pela elevada prevalência -cerca de $20 \%$ da população adulta- como também pela acentuada parcela de hipertensos não diagnosticada, ou não tratada de forma adequada, ou ainda pelo alto índice de abandono ao tratamento. Acarreta graus variáveis de incapacidades e diminuição na expectativa de vida do hipertenso, principalmente devido à insuficiência cardíaca e/ou à insuficiência vascular cerebral, coronariana e renal (14). A HA constitui-se em uma das doenças que pode estar relacionada ao trabalho que tem de ser abordada pelo setor saúde de forma integral e integrada.

Processos infecciosos ocuparam o quarto lugar. O risco de contrair doenças provocadas por esses agentes é maior para os trabalhado- res da área de saúde do que para a população em geral, devido ao contato direto estabelecido com pacientes infectados e/ou vetores transmissores de moléstias infecciosas (15). Dentre as queixas relacionadas aos processos infecciosos, foram mencionadas as amigdalites, múltiplos abcessos pelo corpo, micoses, otites, conjuntivites e herpes. Sabe-se, por exemplo, que o uso do estetoscópio por várias pessoas, sem a higienização adequada após cada uso pode favorecer a presença de otite entre os trabalhadores da saúde. Gotículas de secreções expelidas por pacientes em contato com a mucosa oral ou conjuntiva também podem explicar a presença de amigdalites e conjuntivites nos trabalhadores (16, 17).

Distúrbios do sono aparecem em quinto lugar. Essas alterações são sintomas preocupantes para os trabalhadores em turnos, que terão um bom desempenho profissional se iniciarem seus trabalhos descansados. A fadiga após longos períodos de trabalho sem intervalos pode comprometer tanto o desempenho físico como mental dos trabalhadores em situação de inversão de turnos laborais. O trabalho realiza-se numa fase de desativação psicossomática e na situação de descanso, provocando uma inversão dos ritmos biológicos e interferindo na dessincronização entre ciclo sono-vigília e ciclo dia/ noite, gerando uma sintomatologia de distúrbios do sono, fadiga, alterações de humor, transtornos digestivos, neuropsicológicos, diminuição da motivação para o trabalho, sentimento de exclusão da vida social e perturbação da convivência familiar e da saúde, diminuindo os estímulos auditivos e visuais, sendo observada uma maior frequência de acidentes principalmente no turno da noite (18). Esse fato para a enfermagem é essencial, por se tratar de uma profissão, cujo cuidado ao ser humano não permite erros.

Outras queixas, ocupando o sexto lugar em frequência, são representadas por edema de MMII, febre sem diagnóstico definido e inapetência. A média de queixas de altera- 
ções de saúde encontrada neste estudo, por trabalhador, foi de 2.56 .

Os RO existentes no ambiente laboral dos trabalhadores de enfermagem que atuam em UUE, apresentam-se descritos nas tabelas a seguir.

Tabela 3. Distribuição de riscos ocupacionais identificados no ambiente laboral por 64 trabalhadores de enfermagem que atuam em duas UUE ${ }^{*} 2006(n=301)$.

\begin{tabular}{lrrrrrr}
\hline \multirow{2}{*}{ Riscos ocupacionais $^{* *}$} & \multicolumn{2}{c}{ UUE1 } & \multicolumn{2}{c}{ UUE2 } & \multicolumn{2}{c}{ TOTAL } \\
& \multicolumn{1}{c}{ f } & \multicolumn{1}{c}{$\%$} & \multicolumn{1}{c}{ f } & \multicolumn{1}{c}{$\%$} & \multicolumn{1}{c}{ f } & \multicolumn{1}{c}{$\%$} \\
\hline Psicossocial & 65 & 48.87 & 85 & 50.60 & 150 & 49.83 \\
Biológico & 43 & 32.33 & 56 & 33.33 & 99 & 32.89 \\
Situações anti-ergonômicas & 19 & 14.28 & 21 & 12.50 & 40 & 13.29 \\
Químico & 3 & 2.26 & 4 & 2.38 & 7 & 2.33 \\
Físico & 3 & 2.26 & 2 & 1.19 & 5 & 2.66 \\
Total & 133 & 100.00 & 168 & 100.00 & 301 & 100.00 \\
\hline
\end{tabular}

${ }^{*}$ UUE: Unidade de Urgência e Emergência

${ }^{*}$ Cada trabalhador informou mais de um RO

Constatou-se que os Riscos Psicossociais foram os mais destacados. Foram identificados e mencionados pelos sujeitos das duas unidades, sendo que os da UUE1 referiram 65 vezes este risco e os da UUE2 85 vezes, totalizando 150 citações. Entre os fatores de Riscos Psicossociais mais mencionados pelos trabalhadores da UUE1, destacam-se a ocorrência de agressões físicas e verbais $(61,54 \%)$ e a falta de segurança no trabalho $(23,08 \%)$.
Para os trabalhadores da UUE2 os fatores mais informados foram agressões físicas e verbais $(63,53 \%)$, falta de segurança no trabalho $(14,12 \%)$ e ambiente de trabalho estressante $(10.58 \%)$.

A Tabela 4 apresenta o detalhamento dos agentes de RO identificados pelos trabalhadores de enfermagem que atuam nas duas unidades estudadas.

Tabela 4. Distribuição dos trabalhadores de enfermagem que atuam na UUE1 ${ }^{*}(n=133)$ e $\mathrm{UUE2}^{* *}$ ( $\mathrm{n}=168$ ), segundo os agentes de riscos ocupacionais ${ }^{* * *}$ identificados, 2006.

\begin{tabular}{|c|c|c|c|c|c|}
\hline \multirow[t]{2}{*}{ Agentes de riscos ocupacionais } & \multicolumn{2}{|c|}{ UUE1 } & \multirow[t]{2}{*}{ Agentes de riscos ocupacionais } & \multicolumn{2}{|c|}{ UUE2 } \\
\hline & f & $\%$ & & f & $\%$ \\
\hline Risco Psicossocial $(n=65)$ & & & Risco Psicossocial $(\mathrm{n}=\mathbf{8 5})$ & & \\
\hline Agressões & 40 & 61.54 & Agressões & 54 & 63.53 \\
\hline Falta de segurança & 15 & 23.08 & Falta de segurança & 12 & 14.12 \\
\hline Sobrecarga de trabalho & 4 & 6.15 & $\begin{array}{l}\text { Ambiente estressante na UPA e durante } \\
\text { transporte de pacientes }\end{array}$ & 9 & 10.58 \\
\hline $\begin{array}{l}\text { Ambiente estressante (contato com a doença e } \\
\text { morte) }\end{array}$ & 3 & 4.61 & Desvio de função & 4 & 4.70 \\
\hline Distúrbio do sono & 1 & 1.54 & Sobrecarga de trabalho & 3 & 3.53 \\
\hline Equipamentos insuficientes e ineficientes & 1 & 1.54 & Tudo & 2 & 2.55 \\
\hline Postura arrogante de pacientes e familiares & 1 & 1.54 & Diversidade de diagnósticos médicos & 1 & 1.48 \\
\hline Total & 65 & 100.00 & Total & 85 & 100.00 \\
\hline Risco biológico $(n=43)$ & & & Risco biológico $(n=56)$ & & \\
\hline Contato com doenças infecciosas & 19 & 44.18 & Contato com doenças infecciosas & 30 & 53.57 \\
\hline
\end{tabular}


Continuaçao Tabela 4.

\begin{tabular}{|c|c|c|c|c|c|}
\hline Contaminação por material perfurocortante & 12 & 27.90 & $\begin{array}{l}\text { Falta de material de trabalho e equipamento de } \\
\text { proteção individual (EPI) }\end{array}$ & 9 & 16.07 \\
\hline Falta de equipamento de proteção individual & 5 & 11.63 & Contato com materiais perfurocortantes & 7 & 12.50 \\
\hline Interligação entre PA e área externa & 2 & 4.65 & $\begin{array}{l}\text { Encaminhamento inadequado do lixo e limpeza } \\
\text { deficiente }\end{array}$ & 3 & 5.36 \\
\hline Presença de bolor nas paredes & 1 & 2.33 & Ambiente tumultuado/pressa & 2 & 3.57 \\
\hline $\begin{array}{l}\text { Limpeza deficiente dos filtros do ar } \\
\text { condicionado }\end{array}$ & 1 & 2.33 & Local inadequado para refeições & 2 & 3.57 \\
\hline Planta física inadequada para limpeza & 1 & 2.33 & Água não potável & 2 & 3.57 \\
\hline Pacientes sem diagnóstico definido & 1 & 2.33 & Presença de bolor nas paredes & 1 & 1.79 \\
\hline Repouso no chão & 1 & 2.33 & & & \\
\hline Total & 43 & 100.00 & Total & 56 & 100.00 \\
\hline Situações Anti-ergonômicas (n=19) & & & Situações Anti-ergonômicas $(\mathrm{n}=\mathbf{2 1})$ & & \\
\hline Área física inadequada/riscos de acidentes & 9 & 47.40 & Planta física inadequada & 7 & 33.34 \\
\hline Macas muito altas & 2 & 10.52 & Levantamento de peso & 6 & 28.57 \\
\hline Repouso no chão & 2 & 10.52 & $\begin{array}{l}\text { Equipamentos inadequados ou falta de } \\
\text { equipamentos }\end{array}$ & 5 & 23.81 \\
\hline $\begin{array}{l}\text { Número insuficiente de trabalhadores/ } \\
\text { sobrecarga de trabalho }\end{array}$ & 2 & 10.52 & Postura corporal inadequada & 2 & 9.52 \\
\hline Levantamento de peso & 2 & 10.52 & Risco de quedas/acidentes & 1 & 4.76 \\
\hline Postura corporal inadequada & 2 & 10.52 & & & \\
\hline Total & 19 & 100.00 & Total & 21 & 100.00 \\
\hline Risco Físico (n=3) & & & Risco Físico (n=2) & & \\
\hline Iluminação inadequada & 2 & 66.67 & Iluminação deficiente & 1 & 50.00 \\
\hline Ruído & 1 & 33.33 & Fiação exposta podendo resultar em incêndio & 1 & 50.00 \\
\hline Total & 3 & 100.00 & Total & 2 & 100.00 \\
\hline Risco Químico (n=3) & & & Risco Químico (n=4) & & \\
\hline Contato com produto esterilizante & 2 & 66.66 & Contato com glutaraldeído & 2 & 50.00 \\
\hline \multirow[t]{2}{*}{ Contato com produtos variados de limpeza } & 1 & 33.33 & Contato com medicamentos diversos & 1 & 25.00 \\
\hline & & & Contato com detergente (material de limpeza) & 1 & 25.00 \\
\hline Total & 3 & 100.00 & Total & 4 & 100.00 \\
\hline
\end{tabular}

* UUE1: Unidade de Urgência e Emergência 1

** UUE2: Unidade de Urgência e Emergência 2

*** Cada trabalhador identificou variados fatores de RO

Várias são as condições que fazem com que as UUE sejam locais particularmente propensos à violência no trabalho, entre elas: longas esperas, quadros clínicos altamente estressantes, ambientes ruidosos e um grande número de pacientes que apresentam riscos para comportamentos violentos como: usuários de drogas e álcool, pessoas com doenças mentais e membros de grupos (19). A situação da violência no trabalho nestas UUE é um quadro bem definido, mas comumente subnotificado.

Os agentes de Risco Biológico aparecem sendo mencionados 43 vezes pelos sujeitos da UUE1 e 56 pelos da UUE2, totalizando 99 citações. Para os trabalhadores da UUE1 os agentes deste risco mais identificados foram o contato com clientes portadores de doenças infectocontagiosas $(44,18 \%)$; a contaminação por material perfurocortante $(27,9 \%)$ e a falta de equipamentos de proteção individual (EPI) (11,63\%). Os sujeitos da UUE2 identificaram como os principais agentes deste risco o contato com portadores de doenças infectocontagiosas $(53,57 \%)$, a falta de materiais de trabalho e EPI $(16,07 \%)$ e o risco de acidente com materiais perfurocortantes $(12,50 \%)$. O Risco Biológico encontra-se, inegavelmente, presente nos ambientes de trabalho da área da saúde e é um dos mais identificados pelos trabalhadores, em relação aos demais riscos. Como o trabalho, na maio- 
ria dos ambientes hospitalares e não hospitalares é arriscado e insalubre, encontrando-se os trabalhadores, muitas vezes, realizando as suas tarefas de modo inadequado, em decorrência do desuso de EPI ou sem condições laborais adequadas, decorrentes da estrutura física inapropriada dos estabelecimentos de assistência a saúde. A Norma Regulamentadora - NR 32 intitulada Segurança e Saúde no Trabalho em Serviços de Saúde, foi criada e pretende corrigir, ao menos, uma parte destas distorções. Esta Norma Regulamentadora - NR tem por finalidade estabelecer as diretrizes básicas para a implementação de medidas de proteção à segurança e à saúde dos trabalhadores dos serviços de saúde, bem como daqueles que exercem atividades de promoção e assistência à saúde em geral. Para fins de aplicação desta NR entende-se por serviços de saúde qualquer edificação destinada à prestação de assistência à saúde da população e todas as ações de promoção, recuperação, assistência, pesquisa e ensino em saúde em qualquer nível de complexidade (20).

Faz-se necessário considerar também o agravante de acidentes provocados por agulhas deixadas inadvertidamente em meio a campos cirúrgicos, misturados ao material nos pacotes de curativos, e que terminam por ferir os trabalhadores, os quais, mesmo utilizando EPI, acabam acidentando-se. Embora existam programas educacionais em grande parte das instituições de saúde, o fato é que o problema dos materiais perfurocortantes persiste entre os trabalhadores de enfermagem, de maneira bastante expressiva (21).

As situações anti-ergonômicas estão apresentadas em 40 citações, sendo 19 advindas dos sujeitos da UUE1 e 21 dos da UUE2. Os seguintes agentes destas situações foram identificados pelos sujeitos da UUE1, como sendo área física inadequada/riscos de acidentes $(47,4 \%)$ e com o mesmo percentual $(10,52 \%)$, para cada um, foram mencionados macas muito altas, necessidade de repousar no chão, número insuficiente de trabalha- dores/sobrecarga de trabalho, levantamento de peso e postura corporal inadequada. Para os sujeitos da UUE2, as situações anti-ergonômicas mais identificadas foram planta física inadequada $(33,34 \%)$, levantamento de peso $(28,57 \%)$ e falta e inadequação de equipamentos (23,81\%). Trabalhadores de enfermagem atuam em condições adversas enfrentando a falta de recursos humanos e materiais. É comum a falta de espaço físico adequado, número insuficiente de camas, macas, cadeiras de rodas e de banho (22). A ausência dos recursos materiais surge como um dos maiores sofrimentos no trabalho para a equipe de enfermagem. Desta forma, a escassez de materiais e EPI, conduz à sua constante busca, levando a desgaste e perda de tempo que poderia ser utilizado na assistência aos clientes (13).

Grande parte das agressões à coluna vertebral entre trabalhadores de enfermagem está relacionada aos fatores ergonômicos inadequados de mobiliários; postos de trabalho e equipamentos utilizados nas suas atividades cotidianas e as algias lombares referidas por estes profissionais são na maioria das vezes decorrentes de traumas crônicos repetitivos que envolvem muitos outros fatores, além da manipulação de pacientes (23).

Pesquisadores e organizações têm procurado discutir as técnicas de movimentação e transporte de pacientes dentro de uma estrutura ergonômica e utilizando equipamentos auxiliares (24). No entanto, estes continuam sendo utilizados sem treinamento e em condições inadequadas.

Os agentes de Riscos Químicos são citados sete vezes, sendo, três pelos sujeitos da UUE1 e quatro pelos da UUE2. Na UUE1 foram identificados como agentes, o contato com o produto utilizado para esterilização e desinfecção de materiais, ou seja, o glutaraldeído $(33,33 \%)$ e o contato com produtos variados de limpeza $(66,67 \%)$. Os sujeitos da UUE2 citaram o contato com glutaraldeído duas vezes $(50 \%)$; o contato com medicamentos uma vez (25\%) e com detergente uti- 
lizado na limpeza uma vez (25\%). Este RO é difícil de ser identificado pelos trabalhadores de enfermagem, motivo pelo qual foi pouco citado pelos sujeitos deste estudo, apesar da grande variedade de produtos químicos usualmente encontrados nos ambientes laborais onde atua a equipe de enfermagem. Sabe-se que algumas circunstâncias favorecedoras desse tipo de exposição são: uso prolongado de luvas de látex, o manuseio de detergentes e solventes, a manipulação de drogas antineoplásicas e antibióticos de última geração, a inalação de gases anestésicos, a exposição de vapores de formaldeído e glutaraldeído e aos vapores dos gases esterilizantes, entre outros $(17,25)$.

Os agentes de Riscos Físicos são citados cinco vezes, sendo três pelos sujeitos da UUE1 e dois pelos da UUE2. Para os trabalhadores da UUE1, tais agentes são representados pela iluminação inadequada $(66,67 \%)$ e ruído (33,33\%); os da UUE2 também mencionaram a iluminação deficiente $(50 \%)$ e o risco de incêndio pela presença de fiações elétricas expostas (50\%). Alguns dos agentes presentes no ambiente das áreas de saúde estão representados pelas radiações ionizantes e não ionizantes, ruídos, vibrações, pressões anormais, temperaturas extremas, eletricidade e iluminação (16).

Tais resultados demonstram a ausência de conhecimento dos trabalhadores de enfermagem sobre os riscos químicos e físicos, a exemplo do que ficou evidenciado em outros estudos $(26,27)$.

\section{CONCLUSÃO}

Trabalhadores de enfermagem que atuam em UUE estão expostos aos vários RO; suas condições de saúde estão comprometidas e possivelmente, algumas das alterações de saúde que apresentam são decorrentes de sua exposição a tais riscos. Estes trabalhadores precisam ser observados de forma mais cautelosa por parte dos seus empregadores, partindo do pressuposto que as faixas salariais são baixas e que este fato está diretamente ligado às excessivas cargas horárias laborais apresentadas. Tal contexto favorece o surgimento das alterações à saúde, que prejudicam tanto o trabalhador como a classe patronal, pois o adoecimento leva-o ao afastamento de suas atividades laborais, prejudicando o andamento do trabalho, acumulando as tarefas não realizadas aos demais empregados.

Trabalhadores necessitam de adequação de sua carga horária, ter compensação justa além de boas condições de segurança e saúde laboral. A presença dos diversos agentes de $\mathrm{RO}$ pode estar diretamente relacionada ao surgimento das alterações de saúde encontradas, necessitando que trabalhadores e empregadores atentem-se para tal e cumpram as normatizações existentes, como por exemplo, aquelas descritas na Norma Regulamentadora 32, objetivando minimizar os fatores de riscos existentes nos ambientes laborais dos estabelecimentos de saúde.

\section{REFERÊNCIAS}

1. Sêcco IAO, Robazzi MLCC, Gutierrez PR, Matsuo T. Acidentes de trabalho e riscos ocupacionais no dia-a-dia do trabalhador hospitalar: desafio para a Saúde do Trabalhador. Rev Espaço para à saúde. 2003; 4 (1): 5-10.

2. Sêcco IAO, Robazzi MLCC. Acidentes de trabalho na equipe de enfermagem de um hospital de ensino do Paraná - Brasil. Cienc. enferm. [revista en la Internet]. 2007; 13(2): 65-78. Hallado em: http://www.scielo.cl/scielo. [Acceso em: 13/04/2010].

3. Conselho Federal de Enfermagem - COFEN. Resolução COFEN - 293, de 21 de setembro de 2004. Rio de Janeiro (Brasil); 2004.

4. Almeida MCP, Rocha SMM. O trabalho 
de enfermagem. $1^{\text {a }}$ ed. São Paulo (Brasil): Corte; 1997.

5. Backes DS, Backes MS, Souza FGM, Erdman AL. O papel do enfermeiro no contexto hospitalar: a visão de profissionais de saúde. Cienc Cuid Saúde. 2008; 7(3): 319-326.

6. Conselho Regional de Medicina do Estado do Ceará. Parecer CREMEC n ${ }^{\circ}$ 14/2001. Ceará. (Brasil); 2001.

7. Guimarães LAM, Grubits S. Série saúde mental e trabalho. Vol. II. 1a ed. São Paulo (Brasil): Casa do Psicólogo; 2004.

8. Cezar ES, Marziale MHP. Problemas de violência ocupacional em um serviço de urgência hospitalar da Cidade de Londrina, Paraná, Brasil. Cad Saúde Pública [revista em la Internet].2006; 22(1): 217221. Hallado em: www.scielo.br/scielo. [Acceso em: 13/04/2010].

9. Mendes R. Patologia do trabalho. Vol II. 2a ed. Rio de Janeiro (Brasil): Atheneu; 2003.

10. Aaker D, Kumar V, Day G. Marketing Research. 7a ed. New York (USA): John Wiley \& Sons New York, 2001.

11. Ministério da Saúde (Brasil), Conselho Nacional de Saúde, Comitê Nacional de Ética em Pesquisa com Seres Humanos. Resolução 196, de 10 de outubro de 1996: diretrizes e normas regulamentadoras de pesquisa envolvendo seres humanos. Brasília (DF); 1996.

12. Santos LSC, Girardello EB. Demandas de atenção do enfermeiro no ambiente de trabalho. Rev Latino-Am. Enfermagem. 2007; 15(1): 27-33.

13. Lunardi WDF. Prazer e sofrimento no trabalho: contribuições à organização do processo de trabalho de enfermagem. Rev. bras. enferm. 1997; 50 (1): 77-92.

14. Silva JLL, Souza SL. Fatores de risco para hipertensão arterial sistêmica versus estilo de vida docente. Revista Eletrônica de Enfermagem. 2004; 6(3): 330-335 .

Hallado em: http://www.fen.ufg.br/revista/revista6_3/pdf/03_Original.pdf
[Acceso em: 13/04/2010].

15. Couto OFM, Pedroso ERP. Doenças infecciosas e parasitárias relacionadas com o trabalho. In: Mendes R. (org). Patologia do trabalho: atualizada e ampliada. Vol. II $2^{\text {a }}$ ed. Rio de Janeiro (Brasil): Atheneu; 2003.

16. Takeda E, Robazzi MLCC, Lavrador MAS. Risco ocupacional de adquirir tuberculose entre trabalhadores de enfermagem hospitalar. Rev. bras. enferm. 2001; 54 (3): 456-465.

17. Bulhões I. Riscos do trabalho de enfermagem. $2 \mathrm{a}$ ed. Rio de Janeiro (Brasil): Folha Carioca; 1994.

18. Almondes KM, Araújo JF. Padrão do ciclo sono-vigília e sua relação com a ansiedade em estudantes universitários. Estud. psicol. 2003; 8 (1): 37- 43.

19. Racette K. Violence in the workplace. Radiol Technol. 2001; 72 (4): 329-337.

20. Robazzi MLCC, Barros Junior J. Propuesta Brasilenã de Normatización para los trabajadores de la salud. Cienc. enferm. 2005; 11(2): 11-15.

21. Trevisan MJ, Robazzi MLCC, Garanhani ML. Sentimentos de prazer entre enfermeiros de unidades de terapia intensiva. Cienc. enferm. [revista en la Internet]. 2009; 15(3): 45-53. Hallado en: http:// www.scielo.cl/scielo. [Acceso em : 13/04/2010].

22. Murofuse NT, Marziale MHP. Doenças do sistema osteomuscular em trabalhadores de enfermagem. Rev Latino-Am. Enfermagem. 2005; 13(3): 364-373.

23. Marziale MHP, Carvalho EC. Condições ergonômicas do trabalho da equipe de enfermagem em unidade de internação de cardiologia. Rev Latino-Am. Enfermagem. 1998; 6(1): 99-117.

24. Alexandre NMC, Rogante MM. Movimentação e transferência de pacientes: aspectos ergonômicos e posturais. Rev. esc. enferm. USP. 2000, 34(2): 165-73.

25. Xelegati R, Robazzi MLCC. Riscos químicos a que estão submetidos os traba- 
lhadores de enfermagem: uma revisão de literatura. Rev Latino-Am. Enfermagem. 2001; 11(3): 350-356.

26. Rezende MP, Robazzi MLCC, Sêcco IAO, Suazo SVV. Riscos físicos e sua identificação por auxiliares de enfermagem de hospital de ensino do estado de Minas Gerais, Brasil. Revista de Enfermagem
UFPE On Line. 2009; 3 (3): 152-159.

27. Xelegati R, Robazzi MLCC, Marziale MHP, Haas VJ. Riscos ocupacionais químicos identificados por enfermeiros que trabalham em ambiente hospitalar. Rev Latino-Am. Enfermagem. 2006; 14(2): 214-219. 